\title{
Tissue Self-Affinity and Polarized Light Scattering in the Born Approximation: A New Model for Precancer Detection
}

\author{
Martin Hunter, ${ }^{1, *}$ Vadim Backman, ${ }^{2}$ Gabriel Popescu, ${ }^{1}$ Maxim Kalashnikov, ${ }^{1}$ Charles W. Boone, ${ }^{1}$ Adam Wax, ${ }^{3}$ \\ Venkatesh Gopal, ${ }^{2}$ Kamran Badizadegan, ${ }^{4}$ Gary D. Stoner, ${ }^{5}$ and Michael S. Feld ${ }^{1}$ \\ ${ }^{1}$ G. R. Harrison Spectroscopy Laboratory, Massachusetts Institute of Technology, Cambridge, Massachusetts 02139, USA \\ ${ }^{2}$ Northwestern University, Evanston, Illinois 60208, USA \\ ${ }^{3}$ Department of Biomedical Engineering, Duke University, Durham, North Carolina 27708, USA \\ ${ }^{4}$ Department of Pathology, Massachusetts General Hospital, Boston, Massachusetts 02114, USA \\ ${ }^{5}$ The Ohio State University Comprehensive Cancer Center, Columbus, Ohio 43210, USA
}

(Received 13 October 2005; published 28 September 2006)

\begin{abstract}
Light scattered from biological tissues can exhibit an inverse power law spectral component. We develop a model based on the Born approximation and von Karman (self-affine) spatial correlation of submicron tissue refractive index to account for this. The model is applied to light scattering spectra obtained from excised esophagi of normal and carcinogen-treated rats. Power law exponents used to fit dysplastic tissue site spectra are significantly smaller than those from normal sites, indicating that changes in tissue self-affinity can serve as a potential biomarker for precancer.
\end{abstract}

DOI: 10.1103/PhysRevLett.97.138102

PACS numbers: 87.64.Cc, 42.62.Be, 87.16.-b

Introduction. - Light scattering spectroscopy (LSS) is a technique in which the angular and wavelength dependence of elastically scattered light is used to infer the spatial frequency spectrum of a scattering object. LSS of biological tissue shows significant promise as a noninvasive tool for clinical cancer diagnosis as well as in understanding the genesis of this disease at the subcellular level. Changes in nuclear size and refractive index observed by LSS, for example, have been shown to correlate well with the onset of dysplasia (precancer) in in vivo studies of human epithelial tissues such as colon, bladder, Barrett's esophagus, and oral cavity [1]. In those studies, the approximate sphericity of epithelial cell nuclei and the prominent LSS spectral oscillations associated with their large diameter $(d=5-15 \mu \mathrm{m})$ enabled successful application of Mie theory for modeling the observed backscattered light.

The spectrum of visible light scattered from epithelial cells and tissue, however, often exhibits a scale-invariant, inverse power law component in addition to the oscillations due to large (nuclear) particles. In any particular LSS experiment, the relative strength of the inverse power law component will depend on (i) the type of tissue studied (e.g., how far the basal cell nuclei are from the epithelial surface), and (ii) the choice of LSS scattering parameters (polar and azimuthal scattering angles, $\theta$ and $\phi$, and the wavelength, $\lambda$, and polarization state of the light used in the measurement) [2]. In this Letter, we focus on the physical interpretation of the inverse power law scattering component from cells and tissue, and examine the diagnostic potential of the power law exponent for precancer detection in epithelial tissues.

There are several reports of inverse power law spectral dependence of the reduced scattering coefficient, $\mu_{s}{ }^{\prime}(\lambda) \propto$ $\lambda^{-\gamma}$, of human cells and tissue, with exponents typically in the range $0.5<\gamma<2[3,4]$. The interpretation of these results, however, is varied. In some cases, a narrow Gaussian size distribution of submicron spheres is invoked to account for the inverse power law scattering behavior [3]. Other researchers, however, have assumed instead a broad (nm to $\mu \mathrm{m}$ range) inverse power law size distribution of Mie spheres to account for similar observations $[5,6]$.

In this Letter, we propose a new physical model to account for inverse power law light scattering from biological tissues based on spatially continuous, statistically self-affine fractal fluctuations in tissue refractive index at submicron scales. [A self-affine function $f(x)$ is one whose variance, $S(x)=\left\langle|f(x+a)-f(x)>|^{2}\right\rangle$, scales according to $S(b x) \propto b^{H} S(x)$, where the Hurst parameter, $H$, is limited to the range $0<H<1]$. Fluctuations of this type have been previously observed by phase contrast microscopy in human and mouse tissues [7], as well as in the subnuclear chromatin texture of benign and malignant human breast epithelial cell nuclei [8]. These fluctuations can be described analytically by von Karman correlation functions, which apply to systems with self-affine fractal correlations with an outer scale limiting the range of that behavior, e.g., atmospheric refractive index inhomogeneities describing classical Kolmogorov atmospheric turbulence [7,9]. The novelty of our approach consists of combining a self-affine tissue refractive index model with the Born approximation (i.e., weak scattering regime) to quantitatively account for the spectral dependence of light scattered from submicron features in biological samples. The model is less restrictive than Mie theory, as it allows for continuous spatial fluctuations in the refractive index of submicron tissue inhomogenities and does not require the assumption of discrete, spheroidal scatterers. The model is used to interpret LSS spectra of backscattered light collected from normal and 
dysplastic rat esophagi, in which the inverse power law scattering component is found to be dominant. The diagnostic potential of the resulting spectral parameters is examined, as well as their physical significance to the morphology of precancerous tissue states.

Experimental._LSS spectra were collected from freshly excised esophagus epithelia of normal and carcinogen-treated rats. Tissue samples were illuminated by a collimated, linearly polarized beam of $\mathrm{cw}$ light from a $100 \mathrm{~W}$ xenon arc lamp (illumination spot diameter $=$ $3 \mathrm{~mm}$ ). Light backscattered by a specimen passed through an analyzer and was focused onto the entrance slit of a spectrograph, where $I_{\|}(\lambda)$ and $I_{\perp}(\lambda)$ spectra (polarized parallel and perpendicular to the incident beam, respectively) were collected by a CCD detector over the wavelength range $\lambda=450-710 \mathrm{~nm}$ (horizontal axis) and scattering angle range $175^{\circ}<\theta<180^{\circ}$ at $\phi=0^{\circ}$ (vertical axis) - see Fig. 1. The $I_{\|}(\lambda)$ and $I_{\perp}(\lambda)$ spectra were corrected for stray light background and instrument response, and a residually polarized LSS spectrum, $\Delta I(\lambda)=$ $I_{\|}(\lambda)-I_{\perp}(\lambda)$, then calculated. As shown in Ref. [2], $\Delta I(\lambda)$ discriminates against light diffusely scattered from tissue and is dominated by singly-scattered photons from the topmost epithelial layer (optical density $\tau<1$ ).

Our tissue samples were prepared according to an animal model of carcinogenesis first described by Pozhariski [10], in which the esophagus-specific carcinogen nitrosomethylbenzylamine (NMBA) is subcutaneously administered to Fisher 344 rats, in $0.25 \mathrm{mg} / \mathrm{kg}$ body weight doses, 3 times a week for five weeks. Five rats from each group (control and carcinogen-treated) were sacrificed at 20 weeks postcarcinogen treatment; a $1 \mathrm{~cm}$-long section of esophagus (including stromal layer) was removed from each rat, and LSS spectra were collected from two randomly selected sites from each tissue sample. After the measurements, the spectral collection sites were marked and the specimens were processed for histology. Formalinfixed paraffin embedded sections were cut at $5 \mu \mathrm{m}$, stained with hematoxylin and eosin, and examined microscopi-

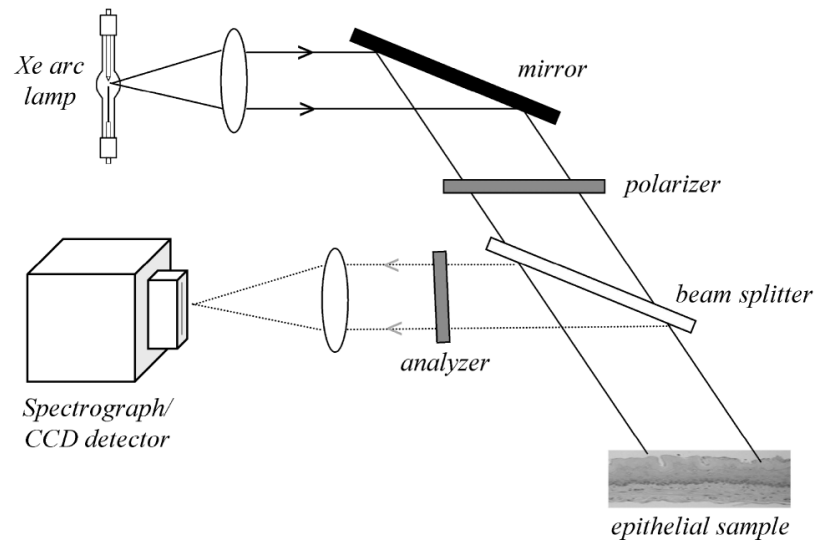

FIG. 1. Schematic diagram of LSS experimental apparatus. cally by an experienced pathologist to confirm the level of dysplasia at each site (Fig. 2). A threefold grading system was implemented (normal, moderately dysplastic, and highly dysplastic) based on our earlier work on precancerous rat esophageal epithelium using low-coherence intereferometry (LCI) [11]. No highly dysplastic sites were observed in this study. Ambiguous registration of pathology and sampled sites in two rat samples (one control, one NMBA treated) resulted in loss of those data sets for analysis.

Model. - In the Born approximation, our LSS spectra are given by the Fourier transform of the tissue refractive index correlation function, $C(\mathbf{r})[12]$ :

$$
\Delta I(\lambda) \propto \lambda^{-4} \int C(\mathbf{r}) \exp (i \mathbf{q} \cdot \mathbf{r}) d^{3} \mathbf{r},
$$

where $C(\mathbf{r})=\frac{k^{2}}{4 \pi} \int\left[n^{2}\left(\mathbf{r}^{\prime}\right)-1\right]\left[n^{2}\left(\mathbf{r}^{\prime}+\mathbf{r}\right)-1\right] d^{3} \mathbf{r}^{\prime}$, with $\mathbf{r}$ the vector correlation distance; $n(\mathbf{r})$ the tissue refractive index; $k=2 \pi / \lambda$; $\mathbf{q}$ the scattering vector with modulus $q=2 k \sin (\theta / 2)$, and $\theta$ the polar scattering angle. The Born approximation holds when the electromagnetic field in the scattering medium is approximately equal to the incident field [12]. This assumption (weak scattering regime) is valid for submicron features in tissue, given the typically small relative refractive index in biological systems $\left(n_{\text {scatterer }} / n_{\text {cytoplasm }}=1.04-1.1[6]\right)$.

In applying Eq. (1) to LSS experiments with cells and tissue, we follow Schmitt and Kumar [7] and employ a form of $C(\mathbf{r})$ given by a von Karman correlation function $[7,9,13]$ :

$$
C(r) \propto\left(\frac{r}{L}\right)^{H} K_{H}\left(\frac{r}{L}\right),
$$

with $r$ the autocorrelation distance, $H$ the Hurst parameter, $L$ the fractal upper scale, and $K_{H}$ a modified Bessel function of the second kind of order $H$. By inserting Eq. (2) into Eq. (1), i.e., by evaluating the three-dimensional Fourier transform of the von Karman correlation function, we obtain the following expression for the observed LSS spectra:

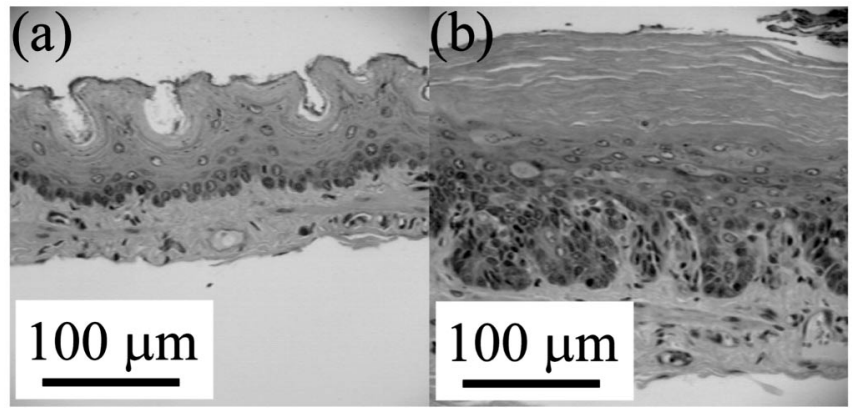

FIG. 2. Hematoxylin and eosin-stained rat esophageal epithelium tissue sections: (a) normal, (b) dysplastic. 


$$
\Delta I(\lambda) \propto \lambda^{-4} \frac{1}{\left[1+(4 \pi L / \lambda)^{2}\right]^{\alpha}},
$$

which we refer to as the fractal-Born approximation. The exponent $\alpha$ is related to the Hurst parameter via $H=\alpha-$ $D_{E} / 2$, with $D_{E}$ the Euclidean dimension of the scattering system (i.e., $D_{E}=1,2$, or 3 for filamentous, sheetlike, or bulk scatterers, respectively) [13,14]. A closely related expression has been derived by Sheppard for predicting angular light scattering intensities from roughened surfaces, albeit under an entirely different set of scattering conditions (Kirchoff approximation) [13].

In the limit where there is no upper bound on the fractal range of correlation lengths $(L \rightarrow \infty)$, the above expression exhibits exact power law behavior, $\Delta I(\lambda) \propto \lambda^{2 \alpha-4}$. Equivalently, under these conditions the von Karman correlation function [Eq. (2)] converges to power law behavior, $C(r) \propto r^{2 \alpha-3}$. In the other extreme, where there exists an upper scale $L \ll \lambda$, the LSS spectrum converges to the Rayleigh limit, $\Delta I(\lambda) \propto \lambda^{-4}$. For intermediate values $(L \sim$ $\lambda / 4 \pi)$, there exists a transition zone where $\Delta I(\lambda)$ will deviate from inverse power law behavior and show curvature on log-log plots of $\Delta I(\lambda)$ vs $\lambda$.

Results. - Six of the eight epithelial sites of NMBAtreated rats were found to be moderately dysplastic. Individual $I_{\|}(\lambda)$ and $I_{\perp}(\lambda)$ spectra from both normal and dysplastic sites showed prominent hemoglobin absorption bands, indicating significant diffuse reflectance contribution from the underlying (vascularized) stromal layer. However, the LSS spectra, $\Delta I(\lambda)$, were clear of such features, showing good discrimination of the LSS technique against diffusely scattered light.

Figure 3 shows typical $\Delta I(\lambda)$ spectra (in log-log scale) obtained at exact backscattering geometry $(\theta=$ $180^{\circ} \pm 0.2^{\circ}$ ) from normal and dysplastic sites, and corresponding fits to these spectra (solid lines) obtained with our

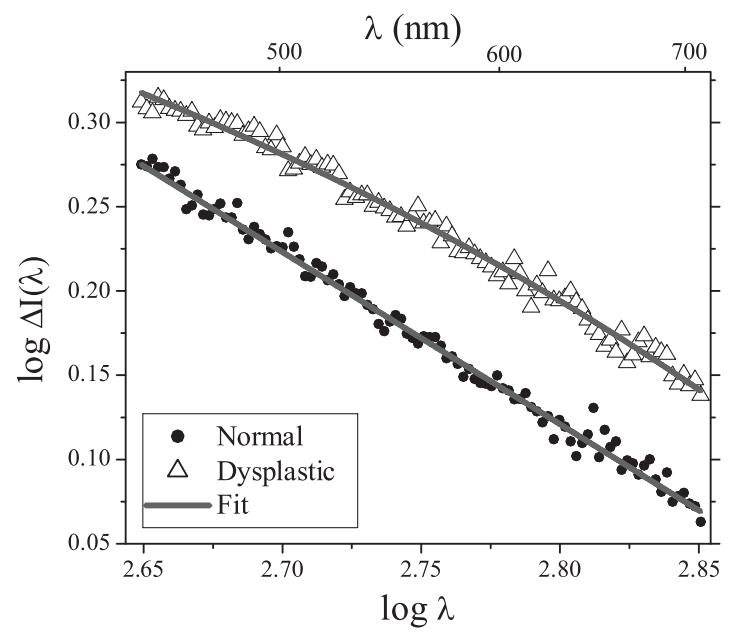

FIG. 3. LSS spectra, in log-log scale, of normal and dysplastic rat esophagus sites. Solid lines are fits to the fractal-Born tissue scattering model. model [Eq. (3)]. The $\Delta I(\lambda)$ spectra are clearly dominated by nonoscillatory behavior, suggesting prominent backscattering contributions from small (submicron), rather than nuclear epithelial structures. Spectra from normal sites were predominantly linear on a log-log scale, indicating inverse power law spectral dependence, $\Delta I(\lambda) \propto \lambda^{-\gamma}$ [with $\gamma=4-2 \alpha$, according to Eq. (3)]. Log-log $\Delta I(\lambda)$ plots from dysplastic sites, on the other hand, consistently showed a small but significant curvature, indicating a reduction in fractal upper scale, $L$, relative to normal tissue sites.

Figure 4 summarizes our experimental results, showing pairs of fractal-Born parameters, $\alpha$ and $L$, obtained from all tissue sites studied and indicating their corresponding histopathological assignment. Significant differences were observed in the power exponents, $\alpha$, extracted from normal and dysplastic sites. The average values of $\alpha$ from normal and dysplastic rat esophagi were $\alpha_{\text {normal }}=1.41 \pm 0.08$ and $\alpha_{\text {dysplastic }}=1.85 \pm 0.07 ; t$-test and Mann-Whitney $u$-test analyses determined this difference to be highly significant $(p<0.00025)$. A clear trend is also observed in the fractal upper scales, $L$, with dysplastic sites tending to have lower values than normal sites. Quantification of this trend, however, was not possible because only a lower bound value for $L$ could be extracted from the majority of normal sites (due to the lack of curvature in their log-log scale LSS plots).

In addition to the dominant power law component, a small residual oscillation $(<1 \%$ relative magnitude) was observable for $\Delta I(\lambda)$ spectra when averaged over a polar angular range $175^{\circ}<\theta<180^{\circ}$, with spectral frequencies significantly larger in dysplastic samples [15]. This is consistent with LCI measurements performed by Wax et al. on similar rat esophagus samples, in which prominent nuclear oscillations as well as inverse power law dependence were observed in the polar angle differential scattering cross sections $[11,16]$. The relatively smaller nuclear

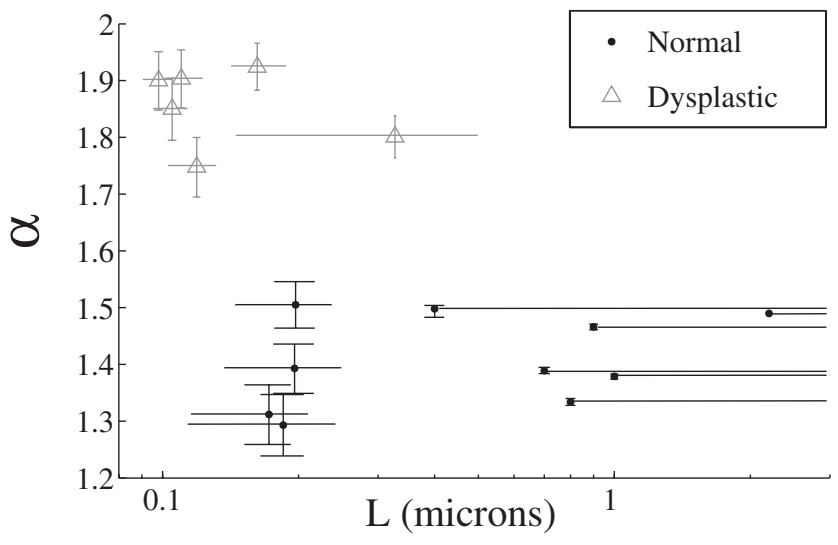

FIG. 4. Plot of fractal-Born parameters $\alpha$ and $L$ for 10 normal and 6 dysplastic sites in rat esophagus LSS study. $L$ values for $L>0.4 \mu \mathrm{m}$ are all lower bounds (shown as one-sided abscissal error bars). 
scattering contribution in our experiments can be accounted for by the fact that LSS is a bulk sampling technique, whereas LCI is capable of optical sectioning and could thus selectively detect light scattered from the nucleus-rich basal cell layer (100 $\mu \mathrm{m}$ below the epithelial surface). In this Letter, we restrict our analysis to the dominant inverse power law LSS component observed in exact backscattering geometry.

Finally, we note that dysplastic samples consistently showed higher signal intensity than normal samples in our spectral window, as seen in Fig. 3. Determination of the direct proportionality constant in Eq. (3) was not possible, however, due to the extreme sensitivity of this parameter to the high variance in $L$ for normal samples.

Discussion. - One major finding from our work is the development of a new spectroscopic tissue parameter, the fractal-Born spectral exponent $\alpha$, for discriminating normal from dysplastic epithelial tissues. This parameter is sensitive to spatial correlations in the tissue refractive index at submicron length scales, thus potentially expanding the diagnostic capability of the LSS technique beyond nuclear size determination [1].

Our results also provide physical insight into the morphology of epithelial tissues, and how this changes with the onset of precancer. The lack of prominent oscillations in the LSS spectra at exact backscattering geometry, in both normal and dysplastic rat esophagi, suggests that submicron tissue inhomogeneities dominate the single backscattering of light from bulk sampling of these tissues. In the majority $(60 \%)$ of normal tissue sites studied here, fits to their LSS spectra by either exact inverse power law or fractal-Born models were statistically indistinguishable, indicating fractal upper scales in these tissues on the order of $L \sim 1 \mu \mathrm{m}$, or higher (Fig. 4, high- $L$ cluster). This result is consistent with the self-affine fractal upper scale values observed by phase contrast microscopy in normal human and mouse tissues $(4 \mu \mathrm{m}<L<10 \mu \mathrm{m})$ [7]. In all dysplastic sites, however, a small but significant curvature in the log-log plots of $\Delta I(\lambda)$ was evident, indicating a reduction in the fractal upper scale of these samples to $L=$ $0.15 \pm 0.09 \mu \mathrm{m}$. We note that some tissue sites graded as normal by histopathology also showed a slight curvature (low- $L$ cluster in Fig. 4), although not as markedly as in dysplastic sites. No histopathological difference was observed, however, between low- $L$ and high- $L$ normally graded tissue sites.

In the fractal-Born tissue scattering model, the selfaffine fractal dimension of tissue, $D$, can be derived from the exponent $\alpha$ [Eq. (3)]. Changes in $\alpha$, therefore, reflect changes in the tissue fractal dimension. However, the value of $D$ is dependent on the Euclidean topological dimension of the scattering object, $D_{E}$, according to: $D=D_{E}+1-$ $H=1.5 D_{E}+1-\alpha[13,14,17]$. Since $0<H<1$, the allowed ranges of $\alpha$ for each topological dimension are: $0.5<\alpha_{1}<1.5,1<\alpha_{2}<2$ and $1.5<\alpha_{3}<2.5$ for $D_{E}=$ 1,2 , and 3, respectively. Therefore, our average values of $\alpha$ obtained from normal and dysplastic rat esophagi, $\alpha_{\text {normal }}=1.41 \pm 0.08$ and $\alpha_{\text {dysplastic }}=1.85 \pm 0.07$, imply that backscattering in normal epithelia was dominated by submicron scatterers of Euclidean dimension $D_{E}=1$ and/ or 2, whereas in dysplastic sites the dominant scatterers had Euclidean dimension $D_{E}=2$ and/or 3. Future work using expanded angular as well as wavelength ranges in LSS spectra will explore the possibility of lifting this ambiguity in derived scatterer topology, $D_{E}$.

The experiments were conducted at the MIT Laser Biomedical Research Center supported by NIH Grant No. RR-02594. Maintenance and carcinogen treatment of Fisher rats were carried out using the support from National Cancer Institute Grant No. CN15011-72, and done in accordance with ethical animal treatment protocol of MIT's Committee on Animal Care. We thank Ronald Nines for technical assistance in handling the Fisher rats during these experiments, and Chung-Chieh $\mathrm{Yu}$ and Condon Lau for useful scientific discussions.

*Electronic address: mhunter@mit.edu

[1] V. Backman et al., Nature (London) 406, 35 (2000).

[2] V. Backman et al., IEEE J. Sel. Top. Quantum Electron. 5 1019 (1999); V. Backman et al., ibid. 7, 887 (2001).

[3] A. M. K. Nilsson et al., Appl. Opt. 37, 1256 (1998); J. R. Mourant et al., ibid. 37, 3586 (1998); F. Bevilacqua et al., ibid. 39, 6498 (2000).

[4] I. S. Saidi, S. L. Jacques, and F. K. Tittel, Appl. Opt. 34, 7410 (1995).

[5] G. Bernard, J. M. Tualle, and S. Avrillier, J. Opt. Soc. Am. A 5, 377 (1996).

[6] J. M. Schmitt and G. Kumar, Appl. Opt. 37, 2788 (1998).

[7] J. M. Schmitt and G. Kumar, Opt. Lett. 21, 1310 (1996).

[8] A. J. Einstein, H.S. Wu, and J. Gil, Phys. Rev. Lett. 80, 397 (1998).

[9] V. I. Tatarskii, Wave Propagation in a Turbulent Medium (Dover, New York, 1967).

[10] K. M. Pozhariski, in Pathology of Tumors in Laboratory Animals, edited by V. S. Turusov, IARC Scientific Pubs. No. 5 (Lyons, France, 1973), Vol. 1, Part 1, p. 87.

[11] A. Wax et al., Cancer Res. 63, 3556 (2003).

[12] M. Born and E. Wolf, Principles of Optics (Cambridge University Press, Cambridge, England, 1999), 7th ed.

[13] C. J. R. Sheppard, Opt. Commun. 122, 178 (1996).

[14] R.F. Voss, in Fundamental Algorithms for Computer Graphics, edited by R. A. Earnshaw (Springer-Verlag, Berlin, 1985).

[15] M. Kalashnikov et al. (to be published).

[16] A. Wax et al., Biophys. J. 82, 2256 (2002).

[17] B. B. Mandelbrot, The Fractal Geometry of Nature (W. H. Freeman, New York, 2000), 19th ed. 